\title{
Direct Observation of Chemical Pressure in Intermetallic Alloys by Scanning Transmission Electron Microscopy
}

\author{
Adedapo A. Oni ${ }^{1}$, Xiahan Sang ${ }^{1}$, Aakash Kumar ${ }^{2}$, Susan B. Sinnott ${ }^{2}$, James M. LeBeau ${ }^{1}$ \\ 1. North Carolina State University, Materials Science and Engineering, Raleigh, USA. \\ 2. University of Florida, Materials Science and Engineering, Gainesville, USA.
}

As solute atoms are added to intermetallic compounds, local chemically induced pressure develops and results in atomic displacements. These distortions play a critical role in defining the mechanical behavior of these materials, for example, by impeding dislocation motion. While indirect methods, such as diffraction determined pair distribution functions, can access the average structure, spatially resolved information is lost. Scanning transmission electron microscopy (STEM), on the other hand, possess excellent spatial resolution, but image distortions often limit the ability to accurately and precisely measure projected crystal structure.

In this talk, we will discuss combining high-angle annular dark-field (HAADF) STEM and density functional theory (DFT) calculations to investigate the correlation between the atom column chemistry and lattice distortion observed in a Ni-based superalloy intermetallic phase. We select a model system, $\gamma^{\prime}$ precipitates in a Ni-Al-Cr superalloy, which adopts the $\mathrm{L}_{2}$ structure (space group $P m-3 m$ ), see Figure 1(a). Using a probe corrected FEI Titan G2 S/TEM, we apply revolving STEM (RevSTEM) to accurately and precisely remove drift distortion [1]. Atomic resolution energy dispersive X-ray spectroscopy (EDS) reveals that Cr preferentially occupies the Al sub-lattice (Figure 1(b)) [3]. Moreover, we will show that lattice strain in Figure 1(c), and crystal distortion (tetragonality), is likely connected to the random fluctuation of $\mathrm{Cr}$ concentration in the Al sub-lattice of the projected STEM images.

To further elucidate the mechanism for relative lattice displacement, relaxation of pure $\mathrm{Ni}_{3} \mathrm{Al}$ and $\mathrm{Ni}_{3}(\mathrm{Al}, \mathrm{Cr})$ supercells are calculated using DFT. These results indicate a relative displacement of the near neighbor $\mathrm{Ni}$ when $\mathrm{Cr}$ is substituted for $\mathrm{Al}$ atoms, as depicted in Figure 2(a). HAADF images are simulated while implementing the static displacement derived from the DFT calculations. The $\mathrm{Ni}_{3}(\mathrm{Al}, \mathrm{Cr})$ supercell was generated by randomly distributing $\mathrm{Cr}$ on $\mathrm{Al}$ sub-lattices, ensuring that the overall concentration of $\mathrm{Cr}$ is approximately the same as the sample. Subsequently, the average Al-Al nearest-like-neighbor (NLN) distances were measured and outlined on the Al sub-lattice in Figure 2(b). From inspection, $\mathrm{Ni}_{3}(\mathrm{Al}, \mathrm{Cr})$ displays a larger distribution of the NLN distances compared to the pure $\mathrm{Ni}_{3} \mathrm{Al}$ of the same thickness. Note, the Cr-rich columns (high intensity) shows larger average NLN distances and the $\mathrm{Al}$ rich columns (low intensity) have lower average NLN distances. The average Al-Al NLN distance plotted against the Al sub-lattice intensity (Figure 2(c)) shows a correlation between the distance and atom column intensity only for $\mathrm{Ni}_{3}(\mathrm{Al}, \mathrm{Cr})$. Within this context, we will examine the correlation between atom column chemistry and displacement for experimental results as a function of solute species and sample thickness. Further, we will discuss how these results can help bridge theoretical and experimental approaches toward understanding the structural chemistry of intermetallic compound alloys [4]. 
References:

[1] X Sang and J. M. LeBeau, Ultramicroscopy 138 (2014) 28-35.

[2] X. Sang, A. A. Oni, J. M. LeBeau, Microsc. Microanal. 20 (2014) 1764.

[3] A. A. Oni et al, Appl. Phys. Lett., 106 (2015) 011601.

[4] The authors acknowledge support from the Air Force Office of Scientific Research (Grant No.

FA9550-12-1-0456). We acknowledge the use of the Analytical Instrumentation Facility (AIF) at North Carolina State University, which was supported by the State of North Carolina and the National Science Foundation.
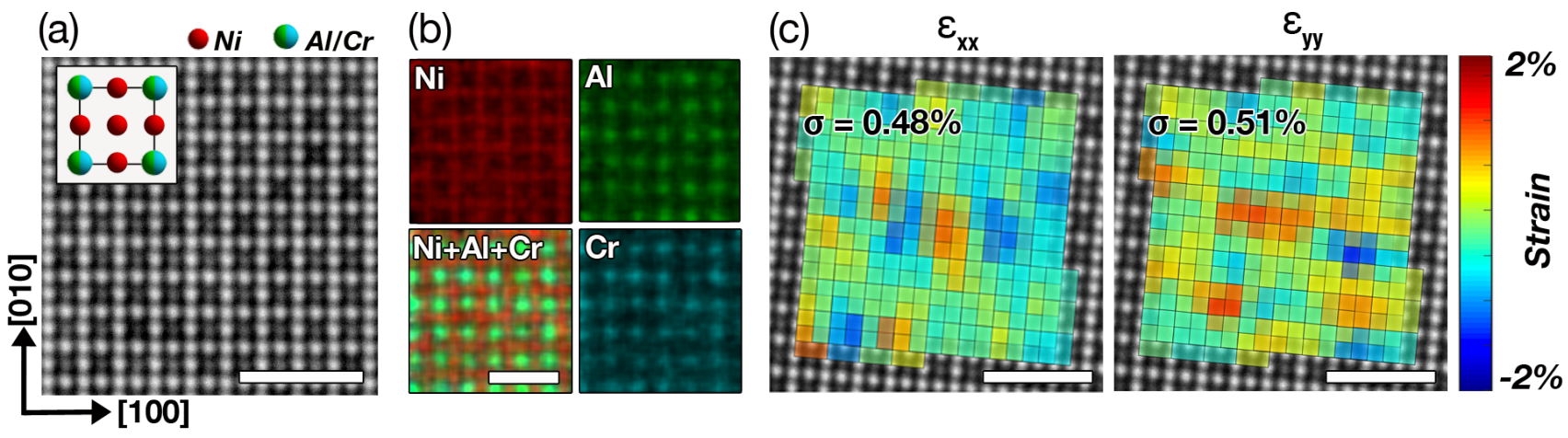

Figure 1. (a) RevSTEM image of $\gamma^{\prime} \mathrm{Ni}_{3}(\mathrm{Al}, \mathrm{Cr}$ ) along $<001>$ with inset showing projected unit cell. (b) Atomic resolution energy dispersive X-ray spectroscopy (EDS) maps. (c) $\varepsilon_{\mathrm{xx}}$ and $\varepsilon_{\mathrm{yy}}$ strain maps. The scale bars represent $1 \mathrm{~nm}$.

(a)

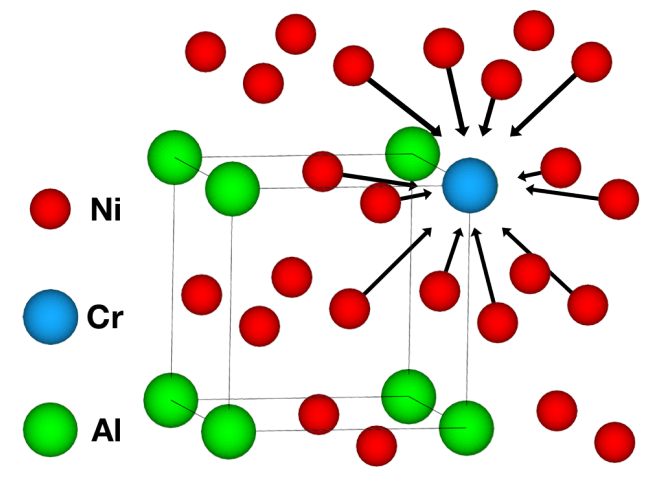

(b)

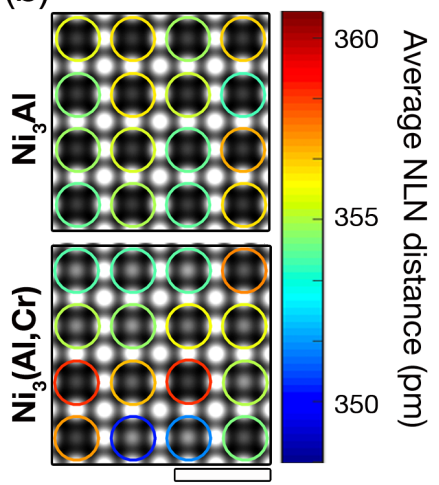

(c)

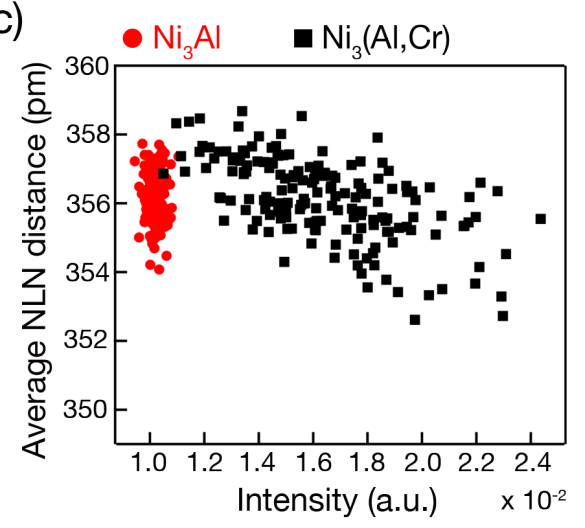

Figure 2. (a) A 2 × 2 × 2 relaxed DFT supercell of the $\mathrm{Ni}_{3}(\mathrm{Al}, \mathrm{Cr})$ structure illustrating the chemical pressure effect of $\mathrm{Cr}$ atoms on the near neighbor $\mathrm{Ni}$ atoms. (b) HAADF-STEM simulations of pure $\mathrm{Ni}_{3} \mathrm{Al}$ and $\mathrm{Ni}_{3}(\mathrm{Al}, \mathrm{Cr})$ with the average NLN distance outlined around each $\mathrm{Al}$ sub-lattice atom column. The intensity of the Ni sub-lattice was clipped to highlight the intensity of the Al sub-lattice. The scale bar represent $500 \mathrm{pm}$. (c) Average Al-Al NLN distances versus atom column intensity from simulated images. 